\section{IAU Symposium}

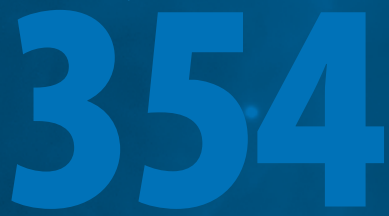

30 June - 6 July, 2019

Copiapo, Chile

\title{
Solar and Stellar
}

Magnetic Fields: Origins and Manifestations

Edited by

Alexander Kosovichev

Klaus Strassmeier

Moira Jardine

ISSN 1743-9213

International Astronomical Union

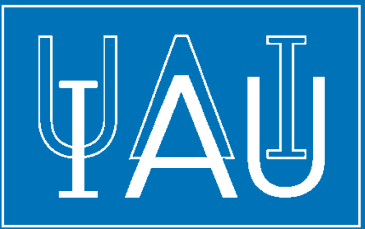

CAMBRIDGE UNIVERSITY PRESS

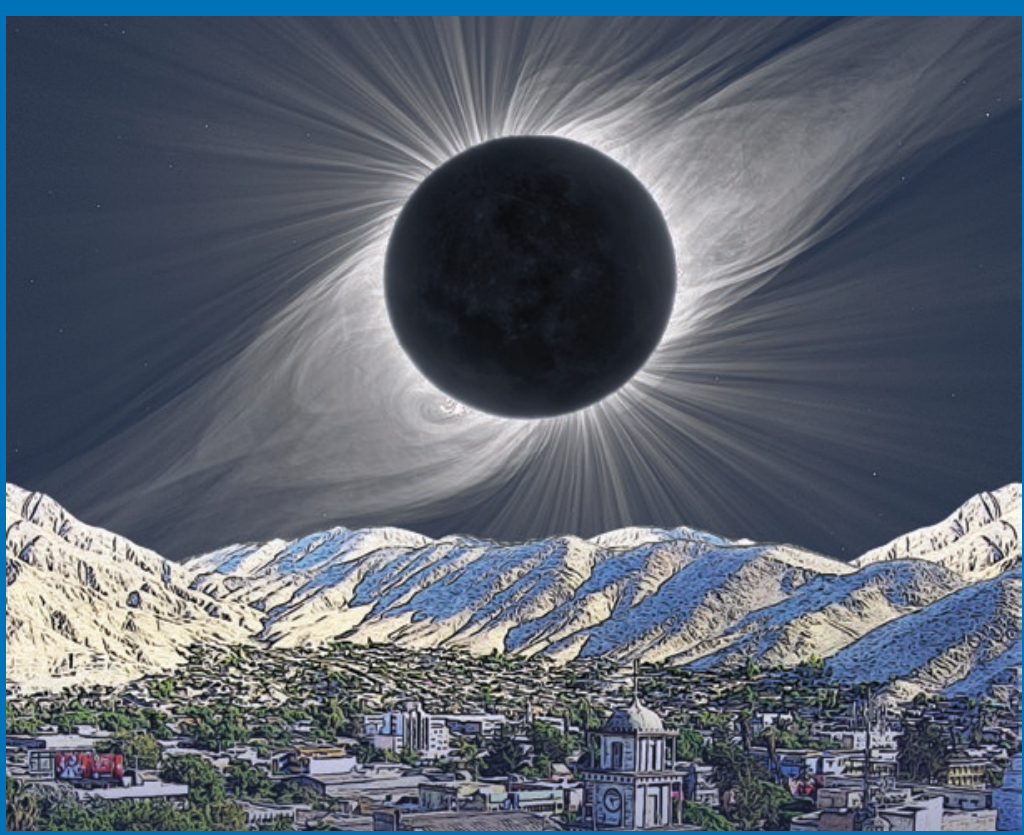




\section{SOLAR AND STELLAR MAGNETIC FIELDS: \\ ORIGINS AND MANIFESTATIONS \\ IAU SYMPOSIUM 354}

IMAGE CREDIT:

The solar eclipse image was obtained on 2 July 2019 near Tres Cruses, Chile, and processed to visualize coronal structures by Miloslav Druckmüller and Peter Aniol. 
IAU SYMPOSIUM PROCEEDINGS SERIES

\author{
Chief Editor \\ MARIA TERESA LAGO, IAU General Secretariat \\ IAU-UAI Secretariat \\ 98-bis Blvd Arago \\ F-75014 Paris \\ France \\ mtlago@astro.up.pt
}

Editor

JOSÉ MIGUEL RODRÍGUEZ ESPINOSA, IAU Assistant General Secretary

\author{
IAU-UAI Secretariat \\ 98-bis Blvd Arago \\ F-75014 Paris \\ France \\ IAU_AGS@iap.fr
}


INTERNATIONAL ASTRONOMICAL UNION

UNION ASTRONOMIQUE INTERNATIONALE

International Astronomical Union

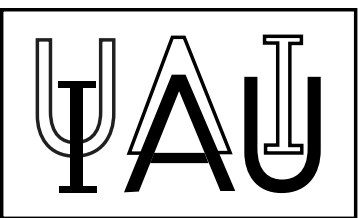

\section{SOLAR AND STELLAR MAGNETIC FIELDS: ORIGINS AND MANIFESTATIONS}

\section{PROCEEDINGS OF THE 354th SYMPOSIUM OF THE INTERNATIONAL ASTRONOMICAL UNION HELD IN COPIAPO, CHILE 30 June-6 July, 2019}

Edited by

ALEXANDER KOSOVICHEV

New Jersey Institute of Technology, USA

KLAUS STRASSMEIER

Leibniz-Institute for Astrophysics Potsdam, Germany

and

MOIRA JARDINE

University of St Andrews, U.K. 
C A M B RIDGE UNIVERSITY PRESS

University Printing House, Cambridge CB2 8BS, United Kingdom

1 Liberty Plaza, Floor 20, New York, NY 10006, USA

10 Stamford Road, Oakleigh, Melbourne 3166, Australia

(C) International Astronomical Union 2020

This book is in copyright. Subject to statutory exception

and to the provisions of relevant collective licensing agreements, no reproduction of any part may take place without

the written permission of the International Astronomical Union.

First published 2020

Printed in Great Britain by Bell \& Bain Ltd, Glasgow

Typeset in System IATEX $2 \varepsilon$

A catalogue record for this book is available from the British Library Library of Congress Cataloguing in Publication data

This journal issue has been printed on $\mathrm{FSC}^{\mathrm{TM}}$-certified paper and cover board. FSC is an independent, non-governmental, not-for-profit organization established to promote the responsible management of the world's forests. Please see www.fsc.org for information.

ISBN 9781108482493 hardback

ISSN 1743-9213 


\section{Table of Contents}

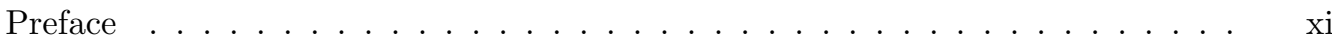

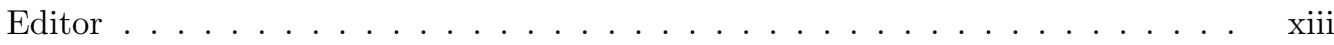

Conference Photograph . . . . . . . . . . . . . . . . . xiv

Participants ...................... $\ldots$ xv

\section{Chapter 1. Total Solar Eclipse of 2019}

Early results from the solar-minimum 2019 total solar eclipse . . . . . . . . . . . 3 Jay M. Pasachoff, Christian A. Lockwood, John L. Inoue, Erin N. Meadors, Aristeidis Voulgaris, David Sliski, Alan Sliski, Kevin P. Reardon, Daniel B. Seaton, Ronald M. Caplan, Cooper Downs, Jon A. Linker, Glenn Schneider, Patricio Rojo and Alphonse C. Sterling

\section{Chapter 2. New observational diagnostics of solar, stellar and interstellar magnetic fields}

Diagnosing coronal magnetic fields with radio imaging-spectroscopy

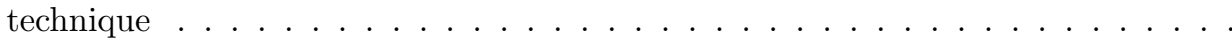

Yihua Yan, Baolin Tan, V. Melnikov, Xingyao Chen, Wei Wang, Linjie Chen, Fei Liu and MUSER Team

Observing the Sun with the Atacama Large Millimeter/submillimeter Array - from continuum to magnetic fields . . . . . . . . . . . . . . . .

Sven Wedemeyer, Mikolaj Szydlarski, Jaime de la Cruz Rodriguez and Shahin Jafarzadeh

Revisiting the building blocks of solar magnetic fields by GREGOR

Dominik Utz, Christoph Kuckein, Jose Iván Campos Rozo, Sergio Javier González Manrique, Horst Balthasar, Peter Gömöry, Judith Palacios Hernández, Carsten Denker, Meetu Verma, Ioannis Kontogiannis, Kilian Krikova, Stefan Hofmeister and Andrea Diercke

Ca II $854.2 \mathrm{~nm}$ spectropolarimetry compared with ALMA and with scattering polarization theory . . . . . . . . . . . . . . . . . . .

J. W. Harvey and SOLIS Team

Diagnosing chromospheric magnetic field through simultaneous spectropolarimetry

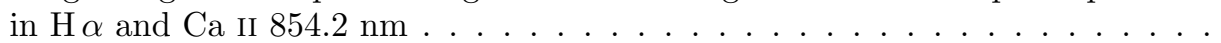

K. Nagaraju, K. Sankarasubramanian and K. E. Rangarajan

The magnetic structure and dynamics of a decaying active region . . . . . . . . .

Ioannis Kontogiannis, Christoph Kuckein,

Sergio Javier González Manrique, Tobias Felipe, Meetu Verma,

Horst Balthasar and Carsten Denker 
Coordinated observations between China and Europe to follow active region $12709 \ldots \ldots \ldots \ldots \ldots \ldots \ldots$

S. J. González Manrique, C. Kuckein, P. Gömöry, S. Yuan, Z. Xu,

J. Rybák, H. Balthasar and P. Schwartz

\section{Chapter 3. Progress in understanding the solar/stellar interior dynamics and dynamos}

Global simulations of stellar dynamos . . . . . . . . . . . . . . . . . 65

G. Guerrero

3D Modeling of the Structure and Dynamics of a Main-Sequence

F-type Star . . . . . . . . . . . . . . . . . . . . . .

Irina N. Kitiashvili and Alan A. Wray

Helioseismic insights into the generation and evolution of the Sun's internal magnetic field . . . . . . . . . . . . . . . . . . . .

Anne-Marie Broomhall and René Kiefer

Resolving Power of Asteroseismic Inversion of the Kepler Legacy Sample . . . . . 107

Alexander G. Kosovichev and Irina N. Kitiashvili

Cycle times of early M dwarf stars: mean field models versus observations . . . .

Manfred Küker, Günther Rüdiger, Katalin Oláh and Klaus Strassmeier

Searching for the cycle period in chromospherically active stars . . . . . . . . . . 120

F. Villegas, R. E. Mennickent and J. Garcés

Are there local dynamo in solar polar region? . . . . . . . . . . . . . . . . . . . . 123

Chunlan Jin

A Clock in the Sun? . . . . . . . . . . . . . . . . . . . . . . . . 127

C. T. Russell, J. G. Luhmann and L. K. Jian

Various scenarios for the equatorward migration of sunspots . . . . . . . . . . . . 134

Detlef Elstner, Yori Fournier and Rainer Arlt

A solar cycle 25 prediction based on 4D-var data assimilation approach . . . . . 138 Allan Sacha Brun, Ching Pui Hung, Alexandre Fournier, Laurène Jouve, Olivier Talagrand, Antoine Strugarek and Soumitra Hazra

Global Evolution of Solar Magnetic Fields and Prediction of Activity Cycles . . . 147 Irina N. Kitiashvili

Solar Open Magnetic Flux Migration Pattern over Solar Cycles . . . . . . . . . . 157 Chia-Hsien Lin, Guan-Han Huang and Lou-Chuang Lee

Probing solar-cycle variations of magnetic fields in the convection zone using meridional flows . . . . . . . . . . . . . . . . . . . Chia-Hsien Lin and Dean-Yi Chou

\section{Chapter 4. Stellar rotation and magnetism}

Magnetic field evolution in solar-type stars

Axel Brandenburg 
Magnetic field and prominences of the young, solar-like, ultra-rapid rotator AP $149 \ldots \ldots \ldots \ldots \ldots \ldots \ldots$

Tianqi Cang, Pascal Petit, Colin Folsom and Jean-Francois Donati

Dipolar stability in spherical simulations: The impact of an inner

Bonnie Zaire and Laurène Jouve

A large rotating structure around AB Doradus A at VLBI scale . . . . . . . . . . 189

J. B. Climent, J. C. Guirado, R. Azulay and J. M. Marcaide

The impact of magnetism on tidal dynamics in the convective envelope of low-mass stars . . . . . . . . . . . . . . . . . . .

A. Astoul, S. Mathis, C. Baruteau, F. Gallet, A. Strugarek,

K. C. Augustson, A. S. Brun and E. Bolmont

The rotation of low mass stars at 30 Myr in the cluster NGC 3766

Julia Roquette, Jerome Bouvier, Estelle Moraux, Herve Bouy,

Jonathan Irwin, Suzanne Aigrain and Régis Lachaume

\section{Chapter 5. Role of magnetic fields in solar and stellar variability}

Possible evidence for a magnetic dynamo in hot Algols .

R. E. Mennickent, J. Garcés, G. Djurašević, G. Rojas, D. Schleicher and $S$. Otero

New Candidates for Chromospherically Young, Kinematically Old Stars . . . . .

Eduardo Machado Pereira and Helio J. Rocha Pinto

The dynamo-wind feedback loop : Assessing their non-linear interplay . . . . . .

Barbara Perri, Allan Sacha Brun, Antoine Strugarek and Victor Réville

Statistical analysis of geomagnetic storms and their relation with the solar cycle . . . . . . . . . . . . . . . . . . . . . .

Paula Reyes, Victor A. Pinto and Pablo S. Moya

Examining the optical intensity and magnetic field expansion factor in the open magnetic field regions associated with coronal holes . . . . . . . . . . . .

Chia-Hsien Lin, Guan-Han Huang and Lou-Chuang Lee

Solar oblateness \& asphericities temporal variations: Outstanding some unsolved issues

Jean P. Rozelot, Alexander G. Kosovichev and Ali Kilcik

\section{Chapter 6. Star-planet relations}

Solar activity influences on planetary atmosphere evolution: Lessons from observations at Venus, Earth, and Mars . . . . . . . . . . . . . . .

J. G. Luhmann

Different types of star-planet interactions

A. A. Vidotto 
Influence of the magnetic field of stellar wind on hot jupiter's envelopes . . . . . 268

Dmitry V. Bisikalo and Andrey G. Zhilkin

Star-planet interaction through spectral lines . . . . . . . . . . . . . . . . 280

C. Villarreal D'Angelo, A. A. Vidotto, A. Esquivel, M. A. Sgró,

T. Koskinen and L. Fossati

From the Sun to solar-type stars: radial velocity, photometry, astrometry and

$\log R_{H K}^{\prime}$ time series for late-F to early-K old stars . . . . . . . . . . . . . 286

Nadège Meunier and Anne-Marie Lagrange

Could star-planet magnetic interactions lead to planet migration and influence

stellar rotation ? . . . . . . . . . . . . . . . . . .

Jérémy Ahuir, Antoine Strugarek, Allan-Sacha Brun, Stéphane Mathis,

Emeline Bolmont, Mansour Benbakoura, Victor Réville and

Christophe Le Poncin-Lafitte

TESS light curves of low-mass detached eclipsing binaries . . . . . . . . . . . 300

Krzysztof G. Hetminiak, Andrés Jordán, Nestor Espinoza

and Rafael Brahm

Tuning in to the radio environment of HD189733b . . . . . . . . . . .

R. D. Kavanagh, A. A. Vidotto, D. Ó Fionnagáin, V. Bourrier, R. Fares,

M. Jardine, Ch. Helling, C. Moutou, J. Llama and P. J. Wheatley

\section{Chapter 7. Formation, structure and dynamics of solar and stellar coronae and winds}

Observational constraints for solar-type stellar winds . . . . . . . . . . . .

Manuel Güdel

Semi-empirical 2D model of the solar corona and solar wind using solar eclipse images: Progress report . . . . . . . . . . . . . . . . . .

Edward C. Sittler Jr. and Linda M. Sittler

Realistic 3D MHD modeling of self-organized magnetic structuring of

the solar corona . . . . . . . . . . . . . . . . . . . .

Irina N. Kitiashvili, Alan A. Wray, Viacheslav Sadykov,

Alexander G. Kosovichev and Nagi N. Mansour

Coherent structures and magnetic reconnection in photospheric and

interplanetary magnetic field turbulence . . . . . . . . . . . . . .

Rodrigo A. Miranda, Abraham C.-L. Chian, Erico L. Rempel and

Suzana S. A. Silva

Analysis of the chromosphere and corona of low-activity early-M dwarfs. . . . . .

Gaetano Scandariato, E. González Álvarez, J. Maldonado,

A. Suárez Mascareño, M. Perger and the HADES collaboration

Reversibility of Turbulent and Non-Collisional Plasmas: Solar Wind . . . . . . .

Belén Acosta, Denisse Pastén and Pablo S. Moya 
Temporal evolution of the velocity distribution in systems described by

the Vlasov equation; Radiation Belts: Analytical and computational results . . .

Abiam Tamburrini C, Iván Gallo-Méndez, Sergio Davis and Pablo S. Moya

On the multifractality of plasma turbulence in the solar wind . . . . . . . . . . . 371

Sebastián Echeverría, Pablo S. Moya and Denisse Pastén

\section{Chapter 8. Mechanisms of flaring and CME activity on the Sun and stars}

The UV/X-ray radiation fields and particle (CME) flows of $\mathrm{M}$ dwarf exoplanet host stars . . . . . . . . . . . . . . . . . . . .

Alexander Brown

Exploring Flaring Behaviour on Low Mass Stars, Solar-type Stars and the Sun . . . . . . . . . . . . . . . . . . . .

L. Doyle, G. Ramsay, J.G. Doyle, P. F. Wyper, E. Scullion, K. Wu and J. A. McLaughlin

Trigger mechanisms of the major solar flares . . . . . . . . . . . . . . . . 392 Shuhong Yang

(Simulating) Coronal Mass Ejections in Active Stars . . . . . . . . . . . . . . . Julián D. Alvarado-Gómez, Jeremy J. Drake, Cecilia Garraffo, Sofia P. Moschou, Ofer Cohen, Rakesh K. Yadav and Federico Fraschetti

Diagnostics of non-thermal-distributions from solar flare EUV line spectra . . . . Elena Dzifčáková, Alena Zemanová, Jaroslav Dudik, and Juraj Lörinčik

Linking radio flares with spots on the active binary UX Arietis . . . . . . . . . . Christian A. Hummel and Anthony Beasley

CME deflections due to magnetic forces from the Sun and Kepler-63 . . . . . . . F. Menezes, Y. Netto, C. Kay, M. Opher and A. Valio

Coronal dimming as a proxy for stellar coronal mass ejections . . . . . . . . . . 426 M. Jin, M. C. M. Cheung, M. L. DeRosa, N. V. Nitta, C. J. Schrijver, K. France, A. Kowalski, J. P. Mason and R. Osten

\section{Chapter 9. Surface magnetic fields of the Sun and stars}

On the properties of the magnetic Chemically Peculiar B, A, and F-type stars . . . . . . . . . . . . . . . . . . . .

Kutluay Yüce, Saul J. Adelman, Diane M. Pyper and Robert J. Dukes

Impact of small-scale emerging flux from the photosphere to the corona: a case study from IRIS . . . . . . . . . . . . . . . . . . . . . . . .

Salvo L. Guglielmino, Peter R. Young, Francesca Zuccarello, Paolo Romano and Mariarita Murabito 
Multi-flux-rope system in solar active regions . . . . . . . . . . . . . . . . . 443

Yijun Hou, Jun Zhang, Ting Li and Shuhong Yang

The 3D structure of the penumbra at high resolution from the bottom of the photosphere to the middle chromosphere . . . . . . . . . . . . . . .

Mariarita Murabito, Ilaria Ermolli, Fabrizio Giorgi, Marco Stangalini, Salvo L. Guglielmino, Shahin Jafarzadeh, Hector Socas-Navarro, Paolo Romano and Francesca Zuccarello

On the Role of Magnetic Fields in an Erupting Solar Filament . . . . . . . . . . 452 Qiao Song, Shuhong Yang and Jing-Song Wang

Fast downflows in a chromospheric filament . . . . . . . . . . . . . . . . . . . . 454 K. Sowmya, A. Lagg, S. K. Solanki and J. S. Castellanos Durán

\section{Chapter 10. Observations of solar eclipses and exoplanetary transits}

Characterization of stellar activity using transits and its impact on

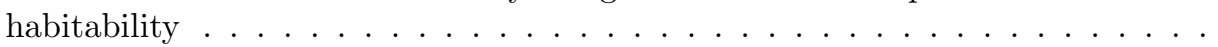

Raissa Estrela, Adriana Valio and Sourav Palit

Discovering the atmospheres of hot Jupiters

P. Wilson Cauley

Sun-as-a-star observations of the 2017 August 21 solar eclipse . . . . . . . . . .

Ekaterina Dineva, Carsten Denker, Meetu Verma, Klaus G. Strassmeier, Ilya Ilyin and Ivan Milic

Solar astrometry with planetary transits . . . . . . . . . . . . . . .

Marcelo Emilio, Rock Bush, Jeff Kuhn and Isabelle Scholl

Author Index . . . . . . . . . . . . . . . . . . . . 


\section{Preface}

Recent observational results from space and ground-based telescopes have convincingly demonstrated that the progress in our understanding of how magnetic fields are generated, how they emerge from the interior, organize in active regions, and cause powerful eruptions can be achieved only by developing a unified approach from relationships between solar and stellar magnetism. Developing a general synergy of solar and stellar astronomy is essential for solving grand-challenge problems like the primary mechanisms of magnetic activity and its impact on planetary atmospheres. An important key issue is that the same or similar phenomena occur on the Sun and other stars under different conditions (different mass, age, metallicity, rotation rate, etc.). Studying these similarities and differences helps to uncover the underlying physical mechanisms of magnetic activity, its evolution in time, and its impacts on planetary environments.

The Proceedings presents recent results and discussions of new emerging topics that include magnetic field diagnostics using high-resolution observation; initial data from ALMA, Chinese Radio Spectroheliograph and other instruments; detection of stellar magnetospheres; detailed mapping of the magnetic fields on the surface of stars using new unique instrumentation, such as the PEPSI spectrograph that provided first highresolution spectropolarimetry with a $12 \mathrm{~m}$ telescope. The new observations stimulate comparisons of solar and stellar results, and advance our understanding of how surface magnetic structures and their evolution are related to the generation of magnetic fields by dynamos in solar and stellar interiors.

In this respect, tremendous progress has been achieved from helioseismology and asteroseismology with data from SDO, Kepler, and TESS, as well as from synoptic observations of solar and stellar variability. Discussions of the current long-term trend of declining solar activity and its initial results on the prediction of the next solar cycles are among the hot topics. The new picture of stellar cycles that is emerging from analysis of the Kepler and supporting ground-based spectroscopic data reveals scaling laws and relations that need to be taken into account in solar magnetism studies. Recent theoretical studies based on advanced supercomputer simulations have demonstrated the key role of magnetism for establishing solar and stellar differential rotation laws, and the importance of observational tests to validate theoretical predictions.

One of the puzzles of solar and stellar magnetism is related to the origin of extreme flare events. During the last weak magnetic cycle, the Sun produced some of the strongest flares in the history of observations. This raises questions on how the flare energetics are related to the magnetism of other stars that produce giant superflares, and what physical mechanism drives such extreme events. Another important topic of joint solar-stellar studies is the influence of solar and stellar variability on planetary space environments which become more and more important.

The interest in understanding the role of stellar magnetism in star-planet relations is driven by the need to determine conditions for habitability. In this aspect, the discussion is focused on properties of solar and stellar coronae and winds, and their interactions with planetary magnetospheres. Compared to the solar system, in many recently discovered planetary systems stellar winds are substantially stronger, and planets are much closer to their parent stars. This creates extreme conditions for magnetic interactions and radiation environments, which depend on the state of stellar magnetic activity. The discussion of this renewed old problem, that is beyond traditional studies, raises interest in understanding the broader impacts of magnetic activity on planetary space weather and habitability. 
These Proceedings present recent advances and key problems of solar and stellar magnetic fields and their impact on planetary atmospheres, discussed at the IAU Symposium 354 "Solar and Stellar Magnetic Fields: Origins and Manifestations", from June 30-July 6, 2019. The Symposium was organized in conjunction with the Total Solar Eclipse of July 2, 2019. The opening paper in Chapter 1 presents the initial observational results of this eclipse. Chapter 2 is focused on new observational diagnostics of solar magnetic fields. The progress in understanding the solar and stellar interior dynamics and dynamos is discussed in Chapter 3. Chapter 4 is devoted to investigations of relationships between stellar rotation and magnetism. The role of magnetic fields in solar and stellar variability is discussed in Chapter 5. Star-planet relations are discussed in Chapter 6 . Chapter 7 is focused on the problem of the formation of solar and stellar coronae and winds. The progress in the understanding of solar and stellar flares and coronal mass ejections is presented in Chapter 8. Some key aspects of magnetic field structures and dynamics on the surface of the Sun and other stars are described in Chapter 9. The final Chapter 10 discusses the role of observations of solar eclipses and exoplanetary transits for characterization of solar and stellar activity and its impacts on the habitability of exoplanets.

The Symposium was organized in close cooperation and support of the University of Atacama, other Chilean universities, as well as of local authorities of the city of Copiapo. In particular, we thank Luis Campusano, Natalie Huerta, Pablo Moya, Lorenzo Morelli, Priscilla Guerrero, and Giovanni Leone for their support, enthusiasm, dedication, and hard work that made the IAUS 354 such a success $\dagger$.

$\dagger$ A previous version of this statement failed to include a number of key individuals. This has been updated to accurately reflect their contribution and a corrigendum published. 


\section{Editor}

Alexander Kosovichev

New Jersey Institute of Technology, USA

Klaus Strassmeier

Leibniz-Institute for Astrophysics Potsdam, Germany

Moira Jardine

University of St Andrews, U.K.

\section{Organizing Committee}

\section{Scientific Organizing Committee}

Alexander Kosovichev (co-chair)

Klaus Strassmeier (co-chair)

Moira Jardine (co-chair)

Pablo Moya (co-chair)

Mauro Barbieri

Alfio Bonanno

Dmitry Bisikalo

Elisabete de Gouveia Dal Pino

Margit Haberreiter

Ahmed Abdel Hady

Elena Khomenko

Tetsuya Magara

Nadege Meunier

Marina Stepanova

Sergio Flores Tullian

Santiago Vargas Dominguez

Michael Wheatland

Jun Zhang

\section{Local Organizing Committee}

Luis Campusano (co-chair)

Lorenzo Morelli (co-chair)

Zhong Wang (co-chair)

Jaime Araneda

Mauro Barbieri

Cristobal Espinoza

Giovanni Leone

Ronald Mennickent

Lorenzo Morelli

Pablo Moya

Roberto Navarro

Andreas Reisenegger

Dominik Schleicher

Mario Soto

Sergio Flores Tullian

Alejandro Valdivia

Wei Wang
New Jersey Institute of Technology, USA

Leibniz-Institute for Astrophysics Potsdam, Germany

University of St Andrews, U.K.

Physics Department, U of Chile

Universidad de Atacama, Chile

Catania Astrophysical Observatory, Italy

Institute of Astronomy, Russia

University of São Paulo, Brazil

PMOD/WRC Davos, Switzerland

Cairo University, Egypt

Instituto de Astrofisica de Canarias, Spain

Kyung Hee University, South Korea

University of Grenoble, France

Physics Department, U of Santiago, Chile

Universidad de Atacama, Chile

National University of Colombia, Colombia

University of Sydney, Australia

National Astronomical Observatory, China

Astronomy Department, Universidad de Chile

Universidad de Atacama

NAOC Astronomy Office in Santiago

Physics Department, U of Concepción

Universidad de Atacama

Physics Department, U of Santiago

Universidad de Atacama

Astronomy Department - University of Concepción

Universidad de Atacama

Physics Department, U of Chile

Physics Department, U of Concepción

Astronomy Department, U Católica

Astronomy Department, U of Concepción

Universidad de Atacama

Universidad de Atacama

Physics Department, U of Chile

NAOC Astronomy Office in Santiago 


\section{CONFERENCE PHOTOGRAPH}

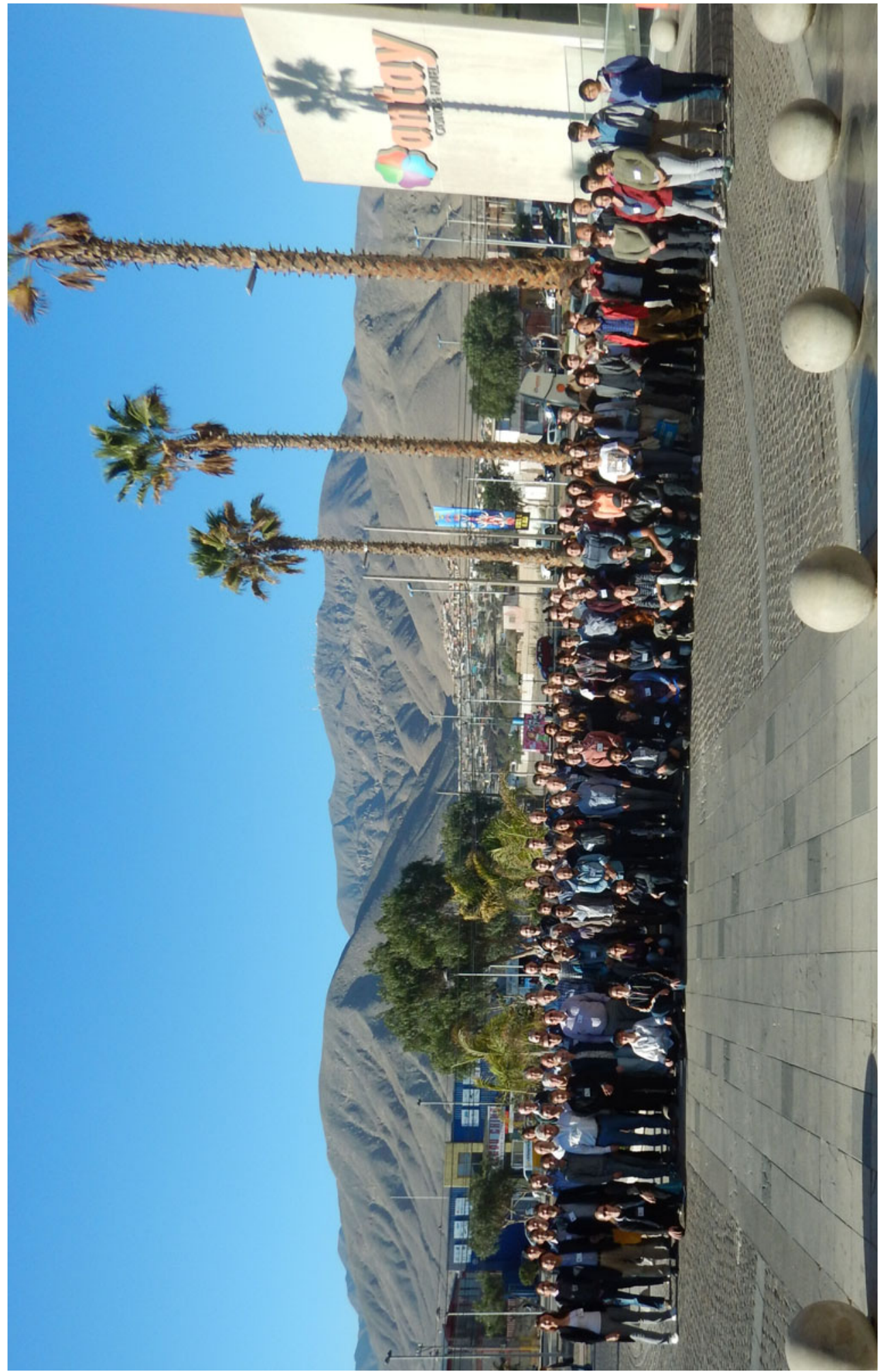


PARTICIPANTS

\begin{tabular}{|c|c|c|}
\hline First Name & Last Name & Country \\
\hline Alicia & Aarnio & USA \\
\hline Belén & Acosta & Chile \\
\hline Jérémy & Ahuir & France \\
\hline Julián David & Alvarado Gómez & USA \\
\hline Louis & Amard & UK \\
\hline Eliana & Amazo-Gomez & Germany \\
\hline Aurélie & Astoul & France \\
\hline Graham & Barnes & USA \\
\hline Luis & Bellot Rubio & Spain \\
\hline Christopher & Bert & USA \\
\hline Lionel & Bigot & France \\
\hline Dmitry & Bisikalo & Russia \\
\hline Veronique & Bommier & France \\
\hline Axel & Brandenburg & Sweden \\
\hline Anne-Marie & Broomhall & UK \\
\hline Alexander & Brown & USA \\
\hline Allan Sacha & Brun & France \\
\hline Paul & Cally & Australia \\
\hline Luis & Campusano & Chile \\
\hline Tianqi & Cang & France \\
\hline Thorsten & Carroll & Germany \\
\hline Paul & Cauley & USA \\
\hline Juan Bautista & Climent Oliver & Spain \\
\hline Manuel & Collados & Spain \\
\hline Simon & Daley-Yates & France \\
\hline Jaime & de la Cruz Rodriguez & Sweden \\
\hline Marc & DeRosa & USA \\
\hline Andrea & Diercke & Germany \\
\hline Ekaterina & Dineva & Germany \\
\hline Lauren & Doyle & UK \\
\hline Elena & Dzifčáková & Czech Republic \\
\hline Sebastian & Echeverrua & Chile \\
\hline Henrik & Eklund & Norway \\
\hline Detlef & Elstner & Germany \\
\hline Marcelo & Emilio & Brazil \\
\hline Raissa & Estrela & USA \\
\hline Adam & Finley & $\mathrm{UK}$ \\
\hline Iván & Gallo & Chile \\
\hline Marta & Garca Rivas & Czech Republic \\
\hline Manuel & Güdel & Austria \\
\hline Gustavo & Guerrero & Brazil \\
\hline Salvo & Guglielmino & Italy \\
\hline John & Harvey & USA \\
\hline Krzysztof & Helminiak & Poland \\
\hline Aleida & Higginson & USA \\
\hline Yijun & Hou & China \\
\hline Christian & Hummel & Germany \\
\hline Patryk & Iwanek & Poland \\
\hline Moira & Jardine & UK \\
\hline Meng & Jin & USA \\
\hline Chunlan & Jin & China \\
\hline Christoffer & Karoff & Denmark \\
\hline Robert & Kavanagh & Ireland \\
\hline Elena & Khomenko & Spain \\
\hline Irina & Kitiashvili & USA \\
\hline
\end{tabular}




\begin{tabular}{|c|c|c|}
\hline First Name & Last Name & Country \\
\hline Lucia & Kleint & Germany \\
\hline Ioannis & Kontogiannis & Germany \\
\hline Kelly & Korreck & USA \\
\hline Alexander & Kosovichev & USA \\
\hline Matthieu & Kretzschmar & France \\
\hline Sowmya & Krishnamurthy & Germany \\
\hline Nagaraju & Krishnappa & India \\
\hline Christoph & Kuckein & Germany \\
\hline Manfred & Küker & Germany \\
\hline Błażej & Kuźma & Poland \\
\hline Zhi-Chao & Liang & Germany \\
\hline Chia-Hsien & Lin & Taiwan \\
\hline Joe & Llama & USA \\
\hline Janet & Luhmann & USA \\
\hline Eduardo & Machado-Pereira & Brazil \\
\hline Fernando & Marques & Brazil \\
\hline Ralph & McNutt & USA \\
\hline Amber & Medina & USA \\
\hline Jorge & Melendez & Brazil \\
\hline Fabian & Menezes & Brazil \\
\hline Ronald & Mennickent & Chile \\
\hline Nadege & Meunier & France \\
\hline Andrea & Miglio & UK \\
\hline Rodrigo & Miranda & Brazil \\
\hline David & Montes & Spain \\
\hline Alberto Jose & Morales-Rodriguez & Costa Rica \\
\hline Roberta & Morosin & Sweden \\
\hline Pablo & Moya & Chile \\
\hline Mariarita & Murabito & Italy \\
\hline Aimee & Norton & USA \\
\hline Dualta & O Fionnagain & Ireland \\
\hline Carlos & Orquera-Rojas & Chile \\
\hline Mayukh & Panja & Germany \\
\hline Jay & Pasachoff & USA \\
\hline Barbara & Perri & France \\
\hline Pascal & Petit & France \\
\hline Alexander & Pietrow & Sweden \\
\hline Geisa & Ponte & Brazil \\
\hline Renzo & Ramelli & Switzerland \\
\hline Paula & Reyes & Chile \\
\hline Alexis Eder & Rodríguez Quiros & Peru \\
\hline Bonnie & Romano Zaire & France \\
\hline Julia & Roquette & UK \\
\hline Jean Pierre & Rozelot & France \\
\hline Christopher & Russell & USA \\
\hline Aoife Maria & Ryan & Ireland \\
\hline Antonia & Savcheva & USA \\
\hline Gaetano & Scandariato & Italy \\
\hline Caius & Selhorst & Brazil \\
\hline Douglas & Silva & Brazil \\
\hline Javier & Silva & Chile \\
\hline Edward & Sittler & USA \\
\hline Sami & Solanki & Germany \\
\hline Qiao & Song & China \\
\hline Klaus & Strassmeier & Germany \\
\hline Antoine & Strugarek & France \\
\hline Alejandro & SuárezMascareño & Spain \\
\hline
\end{tabular}




\begin{tabular}{lll}
\hline First Name & Last Name & Country \\
\hline Ambiam & Tamburrini & Chile \\
Javier & Trujillo Bueno & Spain \\
Adriana & Valio & Brazil \\
Aline & Vidotto & Ireland \\
Carolina & Villarreal D'Angelo & Ireland \\
Fabricio & Villegas & Chile \\
Mariangela & Viviani & Germany \\
Maria & Weber & USA \\
Sven & Wedemeyer & Norway \\
Thomas & Wilson & UK \\
Yihua & Yan & China \\
Shuhong & Yang & China \\
Kutluay & Yuce & Turkey \\
Tomas & Zurita & Chile \\
\hline
\end{tabular}

